\title{
Do Intra-Articular Corticosteroid Injections Prior to Total Knee Arthroplasty Increase Postoperative Complication Rates: A Retrospective Review
}

\section{David Thomas Rhode}

University of Illinois at Chicago College of Medicine

Matthew Alan Siegel ( $\triangle$ Matt.Siegel101@gmail.com )

University of Illinois at Chicago College of Medicine https://orcid.org/0000-0003-1226-3513

\section{Elan Volchenko}

University of Illinois at Chicago College of Medicine

\section{Michael John Patetta}

University of Illinois at Chicago College of Medicine

\section{Garrett R. Schwarzman}

University of Illinois at Chicago College of Medicine

\section{Mark H. Gonzalez}

University of Illinois at Chicago College of Medicine

\section{Research article}

Keywords: Periprosthetic Joint Infection, Total Knee Arthroplasty, Corticosteroid Injection, Intra-articular Injection, Pain Control, Preoperative Management

Posted Date: May 14th, 2020

DOl: https://doi.org/10.21203/rs.3.rs-27904/v1

License: (c) (i) This work is licensed under a Creative Commons Attribution 4.0 International License. Read Full License 


\section{Abstract}

Background:There is conflicting literature suggesting that intra-articular corticosteroid injections before total knee arthroplasty (TKA) may lead to an increase in the rate of postoperative complications, specifically periprosthetic joint infection (PJI). Thus, this systematic review of all TKAs performed at a large, urban hospital will add valuable evidence to help guide future patient care.

Methods: After exclusion criteria, we retrospectively reviewed 417 patients who received a TKA from a group of fellowship-trained orthopaedic surgeons between 2009 to 2016 at a singleacademic medical center. Minimum follow-up time was oneyear. Patients were separated into two groups: those who received a preoperative intra-articular corticosteroid injection and those who did not receive an injection. Subgroups were created based on the timing of their most recent preoperative injection: 0-3 months, 3-6 months, 6-12 months, 12+ months, and an unknown time period. Postoperative outcomes for PJI, revision TKA, and manipulation under anesthesia (MUA)were analyzed via a chi-square test.

Results: No statistically significant postoperative differences were observed between groups: PJI ( $p$-value $=0.904)$, revision TKA ( $p$-value= 0.206$)$, and MUA ( $p$-value= 0.163$)$. The temporal subgroups also failed to demonstrate a statistically significant result: PJI ( $p$-value $=0.348)$, revision TKA ( $p$-value $=0.701)$, and MUA (p-value= 0.512).

Conclusions: This study revealed no absolute or temporal association between preoperative, intraarticular corticosteroid injections and complications after TKA. Because these injections are a commonly used treatment modality prior to TKA, further studies should be conducted on a nationwide basis to draw more concrete conclusions.

\section{Background:}

Osteoarthritis is a debilitating condition, causing mild to moderate disability in up to $10 \%$ of the over-55 population[1]. Current management options include physical therapy to strengthen muscles surrounding the joint, weight loss to reduce forces acting on the joint, pain medications, various intra-articular injections, and surgery[2,3]. Before operative treatments, physicians are encouraged to use less invasive methods to alleviate the pain associated with osteoarthritis. In this scenario, corticosteroid injections are a common treatment modality that may provide short-term relief of osteoarthritis pain[4,5].

While many patients feel they benefit from corticosteroid injections, this form of treatment does not come without concern. The American Academy of Orthopedic Surgeons, in their updated clinical guidelines for the management of osteoarthritis, lowered their recommendations for the use of intraarticular corticosteroid injections. They no longer offer any recommendation for or against them, citing insufficient evidence[6]. In addition, there is speculation that intra-articular corticosteroid injections before total knee arthroplasty (TKA) lead to higher rates of periprosthetic joint infection (PJI). As approximately $30 \%$ of patients receive an injection prior to TKA, there is a significant need to address the relationship between steroid injections and rate of PJI[7]. However, the available literature on this topic demonstrates 
conflicting evidence. Several studies have reported that preoperative, intra-articular corticosteroid injections lead to an increased rate of PJI[8-11], while other studies have reported no difference in surgical outcomes[12-17]. Currently, there are no clear recommendations regarding the timing of these injections with respect to surgery.

The purpose of this study is to add evidence to the controversial literature by comparing infection rates and other complications in post-TKA patients who received intra-articular corticosteroid injections prior to surgery to those in patients who did not receive injections.

\section{Methods:}

This study comprised of a retrospective review of all patients who received a TKA between 2009 and 2016 by a team of adult reconstruction fellowship-trained orthopaedic surgeons at a large, urban academic training hospital. Patient electronic medical records were gathered by using Current Procedural Terminology (CPT) codes. The Institutional Review Board (IRB) from the Office for the Protection of Research Subjects at an academic institution formally reviewed and approved this study.

\section{Retrospective Chart Review}

Patients were excluded if their operation was a revision of a prior TKA or if they failed to follow up for at least one year. A total of 787 patients received a TKA over this time period. 665 of these patients underwent primary TKA, and 417 patients had adequate follow up.

Demographic information such as age, sex, body mass index (BMI), and diabetic status was recorded for each patient. Data was then collected on the presence and timing of corticosteroid injections in relation to TKA via comprehensive review of patients' preoperative clinic notes. All postoperative clinic notes were subsequently analyzed to determine if there were any complications including PJI, the need for revision, and arthrofibrosis requiring manipulation under anesthesia (MUA). Periprosthetic joint infection was defined using the diagnostic criteria developed by the Musculoskeletal Infection Society (MSIS)[18].

\section{Statistical Analysis}

Patients were first divided into two cohorts: 220 patients who received a preoperative intra-articular corticosteroid injection and 197 who did not. The scaled demographics (age and BMI) of the two groups were compared using Mann-Whitney $U$ testing, as Shapiro-Wilk testing revealed a nonparametric distribution. Categorical demographics (prevalence of diabetes mellitus, sex, and laterality) of the two groups were compared using chi-squared testing. Differences in postoperative outcomes (PJI, revision, and manipulation) between the two groups were also analyzed via chi-squared testing.

The injection group was then further divided into subgroups: those who received their most recent injection within 0-3 months of surgery, within 3-6 months, within 6-12 months, longer than 12 months 
prior to surgery, and within an unknown time period before surgery. Chi-square testing was again performed to test for statistically significant differences between the patients who received injections at these various preoperative time intervals and the control group who did not receive injections.

A binary logistic regression model was also performed, as there were statistically significant differences in age and sex noted between the injection group and the control group. These variables were analyzed in order to determine if they were independent predictors for PJI in this study's patient sample.

Analysis was performed with IBM SPSS (International Business Machines Statistical Package for Social Sciences) Version 26.0.0.1 for Mac. The p-value of a was set to 0.05 .

\section{Results:}

220 patients received an intra-articular corticosteroid injection prior to TKA, while the control group consisted of 197 patients who did not receive intra-articular injections prior to surgery. Mean follow-up time was 3.49 years post-TKA for the injection group and 3.93 years after surgery for the no injection group (range 1.00-10.05 years). No patient demographics showed significant differences apart from age and sex. The control group was significantly older $(61.33 \pm 9.33$ years vs. $59.57 \pm 9.19$ years, $p$-value $=$ $0.034)$ and consisted of significantly more males than the injection group [62 (31.47\%) vs. $42(19.09 \%)$, pvalue $=0.004]($ Table 1$)$.

Table 1

Patient demographics

\begin{tabular}{|c|c|c|c|c|c|c|c|}
\hline & $\mathrm{N}$ & $\begin{array}{l}\text { Follow- } \\
\text { up, } \\
\text { years }\end{array}$ & $\begin{array}{l}\text { Diabetes } \\
\text { Mellitus }\end{array}$ & BMI & Age & Sex & Laterality \\
\hline Control & 197 & $\begin{array}{l}3.93 \\
(2.40)\end{array}$ & $\begin{array}{l}57 \\
(28.93 \%)\end{array}$ & $\begin{array}{l}35.28 \\
(7.69)\end{array}$ & $\begin{array}{l}61.33 \\
(9.33)\end{array}$ & $\begin{array}{l}62 \mathrm{M}(31.47 \%) \\
135 \mathrm{~F}(68.53 \%)\end{array}$ & $\begin{array}{l}99 \mathrm{~L}(50.25 \%), 98 \\
\mathrm{R}(49.75 \%)\end{array}$ \\
\hline Injection & 220 & $\begin{array}{l}3.49 \\
(2.06)\end{array}$ & $\begin{array}{l}58 \\
(26.36 \%)\end{array}$ & $\begin{array}{l}35.61 \\
(7.64)\end{array}$ & $\begin{array}{l}59.57 \\
(9.19)\end{array}$ & $\begin{array}{l}42 \mathrm{M}(19.09 \%) \\
178 \mathrm{~F}(80.91 \%)\end{array}$ & $\begin{array}{l}99 \mathrm{~L}(45.00 \%) \\
121 \mathrm{R}(55.00 \%)\end{array}$ \\
\hline$p$-value & & & 0.558 & 0.867 & 0.034 & 0.004 & 0.283 \\
\hline
\end{tabular}

When comparing the postoperative outcomes of the injection group to the control group, the results were similar. There were no statistically significant differences between groups when studying PJI ( $p$ value $=0.904)$, need for revision ( $p$-value $=0.206$ ), or need for manipulation ( $p$-value $=0.163$ ) (Table 2$)$. 
Table 2

Postoperative outcomes comparing overall groups

\begin{tabular}{|lllll|}
\hline & N & PJI & Revision & Manipulation \\
\hline Control & 197 & $5(2.54 \%)$ & $10(5.08 \%)$ & $19(9.64 \%)$ \\
\hline Injection & 220 & $6(2.73 \%)$ & $18(8.18 \%)$ & $31(14.09 \%)$ \\
\hline p-value & 0.904 & 0.206 & 0.163 \\
\hline \multicolumn{4}{|l}{ Legend: Data presented - positive event (\%) } \\
\hline
\end{tabular}

Analysis of the injection groups by timing of injection also failed to reach statistical significance. Chisquared results of postoperative outcomes featured: $P J I$ ( $p$-value $=0.348)$, need for revision $(p-$ value $=0.701)$, and need for manipulation $(p-v a l u e=0.512)($ Table 3$)$.

Table 3

Postoperative outcomes stratified by the timing of the most recent preoperative injection

\begin{tabular}{|lllll|}
\hline & N & PJI & Revision & Manipulation \\
\hline No injection & 197 & $5(2.54 \%)$ & $10(5.08 \%)$ & $19(9.64 \%)$ \\
\hline 0-3 months & 37 & $0(0.00 \%)$ & $2(5.41 \%)$ & $4(10.81 \%)$ \\
\hline 3-6 months & 43 & $3(6.98 \%)$ & $4(9.31 \%)$ & $8(18.60 \%)$ \\
\hline 6-12 months & 35 & $1(2.86 \%)$ & $4(11.43 \%)$ & $6(17.14 \%)$ \\
\hline 12+ months & 58 & $2(3.45 \%)$ & $5(8.62 \%)$ & $6(10.34 \%)$ \\
\hline Unknown & 47 & $0(0.00 \%)$ & $3(6.38 \%)$ & $7(14.89 \%)$ \\
\hline p-value & 0.348 & 0.701 & 0.512 \\
\hline Legend: Data presented - positive event (\%) \\
\hline \multicolumn{5}{|l}{} \\
\hline
\end{tabular}

Binomial regression of sex $(p$-value $=0.381)$ and age $(p$-value $=0.999)$ failed to reach statistical significance as independent predictors of PJI (Table 4).

Table 4

Binary logistic regression for periprosthetic joint infection outcomes on statistically significant demographic data

\begin{tabular}{|llll|}
\hline Independent Variable & p-value & Odds Ratio & $95 \% \mathrm{Cl}$ \\
\hline Sex & 0.381 & 0.572 & $0.164-1.995$ \\
\hline Age & 0.999 & 1.000 & $0.937-1.067$ \\
\hline
\end{tabular}

\section{Discussion:}


This study was unable to draw any correlation between the preoperative administration of intra-articular corticosteroid injections and an increased rate of postoperative complications of TKA, including PJI. The findings of this study mirror those of two 2014 meta-analyses that also reported no increased risk of postoperative PJI after preoperative corticosteroid injections. However, due to the underpowered and heterogeneous nature of many prior studies, both of these publications cited the need for increased larger-sized studies on the matter[14,15]. Since that time, additional studies seem to display dichotomy between relatively smaller, single center studies[16,17], such as this one, and larger database studies[9,11].

Works by Amin et al. found no significant difference in the infection rates of 783 patients who received an injection and 845 who did not[16]. In a similar manner, Kokubun et al. found no significant difference in complication rates, infection rates, and short-term functional outcomes of 175 patients who received four or more preoperative injections and 267 who received three or less[17].In short, these single-center retrospective chart review studies contained similar patient numbers to our study and also observed no significant association between preoperative intra-articular corticosteroid injections and an increased postoperative infection rate.

Conversely, two larger studies utilizing administrative claims data have demonstrated a link between preoperative intra-articular corticosteroid injections and increased rates of postoperative infection. Cancienne et al. found a statistically significant increase in the rates of postoperative infection among 5,313 patients who had received an injection within three months of surgery and also in 8,919 patients who received an injection between three and six months before surgery compared to 13,650 matched controls who did not receive an injection. They reported no statistically significant increase in infection rate among 8,008 patients who received their injections between six and twelve months before surgery[9]. In another study looking at 29,603 patients with an injection and 54,081 without, Bedard et al. found a statistically significant increased risk of infection with an injection before surgery. When the injection cohort was stratified into time periods, the association persisted for an injection within up to six months of surgery[11].Our study found no association of infection from injections at any time period prior to patients' TKA (Table 3).

Both Cancienne's and Bedard's groups discussed the limitations of these large database studies, which included a dependence on proper coding, a lack of demographic data, and an inability to differentiate the specific site or contents of the injections. The advantage of our smaller, single-centered study is that we were able to address all of these issues.

The control group of this study was both older and contained a greater percentage of male patients. Though age alone has not been shown to be associated with an increased risk of PJI[19], male sex has been identified as a risk factor[20,21]. Despite this, binomial regression found that neither age nor sex were independent risk factors for PJI in this study. Hence, differences in sex between the two cohorts had no significant impact on the outcome of this study. A new meta-analysis including the more recent 
smaller scale studies could potentially overcome these limitations while evaluating a larger, more generalizable population.

\section{Limitations}

The fact that the larger database studies were able to demonstrate an association between injections and infection, while recent smaller scale studies were not, suggests the possibility that an association may indeed exist, but our study lacked the power to detect it. According to Desai et al., a study would need 2,000 patients in each cohort to detect a 50\% increase in infection with a 95\% confidence interval[13]. Though this study may be underpowered, it contains one of the largest samples of clinical (not administrative) data on this topic to date.

The total rate of PJI for patients included in this study was $2.64 \%(11 / 417)$. This is above the estimated rates of $1-2 \%$ following primary TKA[20,22]. However, this value is very likely inflated due to the inclusion criteria of the study. Patients who developed a PJI were much more likely to return to clinic, and therefore all met the one-year follow-up minimum for inclusion. When re-including all patients who underwent primary TKA regardless of follow-up, the rate of PJI drops to $1.65 \%$ (11/665), a value likely closer to the true incidence of PJI at the hospital of study and within the range estimated in the literature.

Also, as seen with most single-center studies, we were only able to detect injections noted within our hospital system. Therefore, injections given by providers outside of the hospital system could have been missed, potentially weakening an association between injections and infection by falsely elevating the control group rate.

\section{Conclusions:}

Our study suggests that intra-articular corticosteroid injections before TKA do not increase the rate of PJI, the need for manipulation under anesthesia, or the need for revision surgery. Therefore, this treatment modality may remain a consideration for short-term pain relief in patients awaiting TKA. Further studies should be conducted to draw definitive conclusions regarding the relationship between preoperative intraarticular corticosteroid injections and postoperative complications after TKA.

\section{List Of Abbreviations:}

Total Knee Arthroplasty (TKA), Periprosthetic Joint Infection (PJI), Body Mass Index (BMI), Current Procedural Terminology (CPT), Institutional Review Board (IRB), Manipulation Under Anesthesia (MUA), Musculoskeletal Infection Society (MSIS),IBM SPSS (International Business Machines Statistical Package for Social Sciences)

\section{Declarations:}




\section{Ethics approval and consent to participate}

The Institutional Review Board (IRB) from the Office for the Protection of Research Subjects at an academic institution formally reviewed and approved this study.Research Protocol Number: 2013-1236

\section{Consent for publication}

Not applicable

\section{Availability of data and materials}

The datasets used and/or analyzed during the current study are available from the corresponding author on reasonable request.

\section{Competing interests}

All authors have no competing interests.

\section{Funding}

Not applicable

\section{Authors' contributions:}

DTR collected and interpreted patient demographics and outcomes data, performed the bulk of the literature search, and was a major contributor in writing the manuscript.MAS collected and interpreted patient demographics and outcomes data, contributed to the literature search, and was a major contributor in writing the manuscript. EV collected and interpreted patient outcomes data and contributed to writing the manuscript.MJP interpreted patient outcomes data and contributed to writing the manuscript.GRS contributed to writing the manuscript.MHG guided and contributed to writing the manuscript.All authors read and approved the final manuscript.

\section{Acknowledgements}

Not applicable

\section{Authors' information}




\section{References:}

1. Peat G, McCarney R, Croft P. Knee pain and osteoarthritis in older adults: a review of community burden and current use of primary health care. Annals of the Rheumatic Diseases. 2001;60:91-7.

2. Scott DL. Guidelines for the Diagnosis, Investigation and Management of Osteoarthritis of the Hip and Knee. J R Coll Physicians Lond. 1993;27:391-6.

3. Hochberg MC, Altman RD, Brandt KD, Clark BM, Dieppe PA, Griffin MR, et al. Guidelines for the medical management of osteoarthritis. Arthritis \& Rheumatism. 1995;38:1541-6.

4. Arroll B, Goodyear-Smith F. Corticosteroid injections for osteoarthritis of the knee: meta-analysis. BMJ. 2004;328:869.

5. Bellamy N, Campbell J, Welch V, Gee TL, Bourne R, Wells GA. Intraarticular corticosteroid for treatment of osteoarthritis of the knee. Cochrane Database of Systematic Reviews [Internet]. 2006 [cited 2019 Jul 16]; Available from: http://www.cochranelibrary.com/cdsr/doi/10.1002/14651858.CD005328.pub2/abstract

6. Jevsevar D. Treatment of Osteoarthritis of the Knee: Evidence-Based Guideline, 2nd Edition. Journal of the American Academy of Orthopaedic Surgeons. 2013;21:571-6.

7. Horne G, Devane P, Davidson A, Adams K, Purdie G. The influence of steroid injections on the incidence of infection following total knee arthroplasty. N Z Med J. 2008;121:U2896.

8. Papavasiliou AV, Isaac DL, Marimuthu R, Skyrme A, Armitage A. Infection in knee replacements after previous injection of intra-articular steroid. The Journal of Bone and Joint Surgery British volume. 2006;88-B:321-3.

9. Cancienne JM, Werner BC, Luetkemeyer LM, Browne JA. Does Timing of Previous Intra-Articular Steroid Injection Affect the Post-Operative Rate of Infection in Total Knee Arthroplasty? The Journal of Arthroplasty. 2015;30:1879-82.

10. Schairer WW, Nwachukwu BU, Mayman DJ, Lyman S, Jerabek SA. Preoperative Hip Injections Increase the Rate of Periprosthetic Infection After Total Hip Arthroplasty. The Journal of Arthroplasty. 2016;31:166-169.e1.

11. Bedard NA, Pugely AJ, Elkins JM, Duchman KR, Westermann RW, Liu SS, et al. The John N. Insall Award: Do Intraarticular Injections Increase the Risk of Infection After TKA? Clin OrthopRelat Res. 2017;475:45-52.

12. Joshy S, Thomas B, Gogi N, Modi A, Singh BK. Effect of intra-articular steroids on deep infections following total knee arthroplasty. Int Orthop. 2006;30:91-3.

13. Desai A, Ramankutty S, Board T, Raut V. Does intraarticular steroid infiltration increase the rate of infection in subsequent total knee replacements? The Knee. 2009;16:262-4.

14. Charalambous $C P$, Prodromidis AD, Kwaees TA. Do Intra-Articular Steroid Injections Increase Infection Rates in Subsequent Arthroplasty? A Systematic Review and Meta-Analysis of Comparative Studies. 
The Journal of Arthroplasty. 2014;29:2175-80.

15. Wang Q, Jiang X, Tian W. Does Previous Intra-Articular Steroid Injection Increase the Risk of Joint Infection Following Total Hip Arthroplasty or Total Knee Arthroplasty? A Meta-Analysis. Med Sci Monit. 2014;20:1878-83.

16. Amin NH, Omiyi D, Kuczynski B, Cushner FD, Scuderi GR. The Risk of a Deep Infection Associated With Intraarticular Injections Before a Total Knee Arthroplasty. The Journal of Arthroplasty. 2016;31:240-4.

17. Kokubun BA, Manista GC, Courtney PM, Kearns SM, Levine BR. Intra-Articular Knee Injections Before Total Knee Arthroplasty: Outcomes and Complication Rates. The Journal of Arthroplasty. 2017;32:1798-802.

18. New Definition for Periprosthetic Joint Infection. The Journal of Arthroplasty. 2011;26:1136-8.

19. Inoue $D$, Xu C, Yazdi H, Parvizi J. Age alone is not a risk factor for periprosthetic joint infection. J Hosp Infect. 2019;103:64-8.

20. Kapadia BH, Berg RA, Daley JA, Fritz J, Bhave A, Mont MA. Periprosthetic joint infection. The Lancet. 2016;387:386-94.

21. Kunutsor SK, Whitehouse MR, Blom AW, Beswick AD, Team I. Patient-Related Risk Factors for Periprosthetic Joint Infection after Total Joint Arthroplasty: A Systematic Review and Meta-Analysis. PLOS ONE. 2016;11:e0150866.

22. 22. Kurtz SM, Ong KL, Lau E, Bozic KJ, Berry D, Parvizi J. Prosthetic Joint Infection Risk after TKA in the Medicare Population. Clin OrthopRelat Res. 2010;468:52-6. 